$$
\begin{aligned}
& 7300 \\
& f l l
\end{aligned}
$$

\title{
SYSTEM CHARACTERIZATION OF POSITIVE REAL CONDITIONS
}

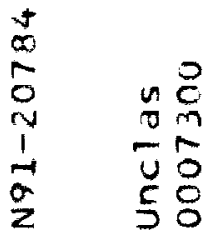

$\frac{9}{0}$

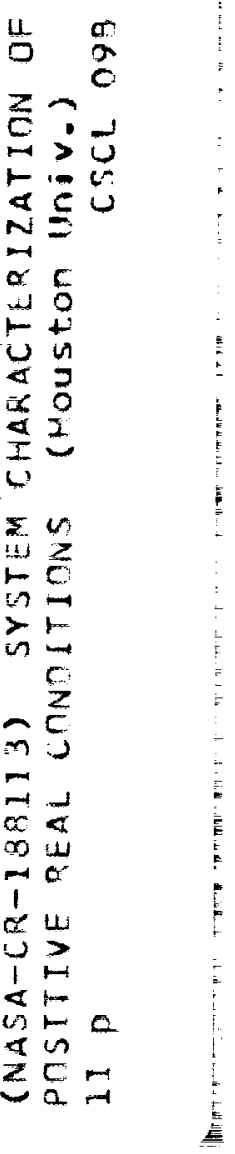

Q. Wang

J.L.Speyer

H. Weiss

University of California, Los Angeles

Cooperative Agreement NCC 9-16

Research Activity No. MS.4

NASA Johnson Space Center

Engineering Directorate

Navigation, Control, \& Aeronautics Divislon

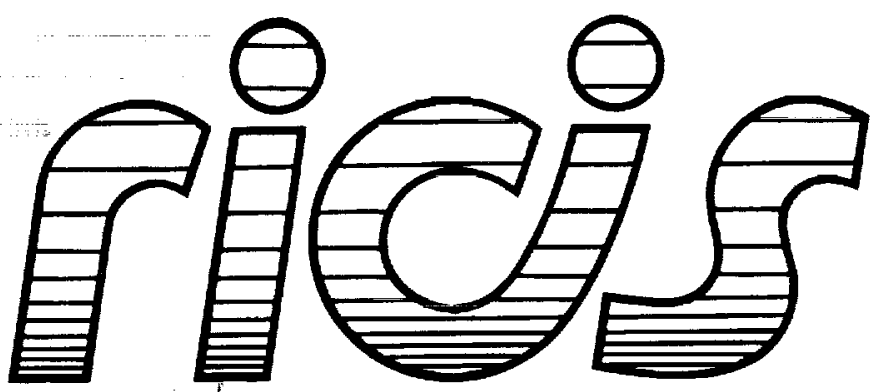

Research Institute for Computing and Information Systems University of Houston - Clear Lake 
The University of Houston-Clear Lake established the Research Institute for Computing and Information systems in 1986 to encourage NASA Johnson Space Center and local industry to actively support research in the computing and information sciences. As part of this endeavor, UH-Clear Lake proposed a partnership with JSC to jointly define and manage an integrated program of research in advanced data processing technology needed for JSC's main missions, including administrative, engineering and science responsibilities. JSC agreed and entered into a three-year cooperative agreement with UH-Clear Lake beginning in May, 1986, to jointly plan and execute such research through RICIS. Additionally, under Cooperative Agreement NCC 9-16, computing and educational facilities are shared by the two institutions to conduct the research.

The mission of RICIS is to conduct, coordinate and disseminate research on computing and information systems among researchers, sponsors and users from UH-Clear Lake, NASA/JSC, and other research organizations. Within UH-Clear Lake, the mission is being implemented through interdisciplinary involvement of faculty and students from each of the four schools: Business, Education, Human Sciences and Humanities, and Natural and Applied Sciences.

Other research organizations are involved via the "gateway" concept. UH-Clear Lake establishes relationships with other universities and research organizations, having common research interests, to provide additional sources of expertise to conduct needed research.

A major role of RICIS is to find the best match of sponsors, researchers and research objectives to advance knowledge in the computing and information sciences. Working jointly with NASA/JSC, $\bar{R} \overline{I C I} \bar{S}$ advises on research needs, recommends principals for conducting the research, provides technical and administrative support to coordinate the research, and integrates technical results into the cooperative goals of UH-Clear Lake and NASA/JSC. 
SYSTEM CHARACTERIZATION OF POSITIVE REAL CONDITIONS 


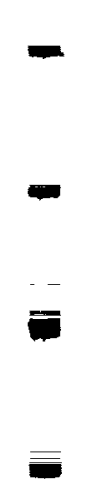

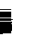

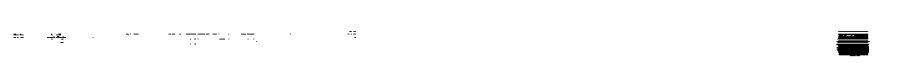

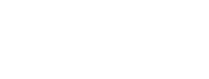

(2)

$\square$

$\bar{\square}$

$\bar{\equiv}$

$\bar{E}$

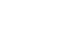

$=$

$=$

$=$

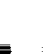

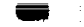

$\bar{\equiv}$

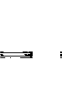

플

E

-

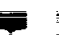

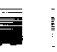

- 


\section{Preface}

This research was conducted under the auspices of the Research Institute for Computing and Information Systems by Q. Wang, J.L. Speyer, and H. Weiss of the University of California, Los Angeles. Dr. A. Glen Houston, Director of RICIS, served as RICIS research representative.

Funding has been provided by Navigation Control \& Aeronautics Division, Engineering Directorate, NASA/JSC through Cooperative Agreement NCC 9-16 between NASA Johnson Space Center and the University of Houston-Clear Lake. The NASA technical monitor for this activity was David Geller, of the Navigation Section, Navigation and Gridance Systems Branch, Navigation Control \& Aeronautics Division, Engineering Directorate, NASA/JSC.

The views and conclusions contained in this report are those of the author and should not be interpreted as representative of the official policies, either express or implied, of NASA or the United States Government. 

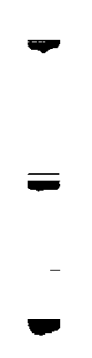

E

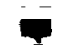

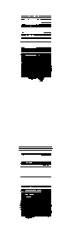

$\equiv$

更

$=$

$\overline{\overline{\bar{\eta}}}$

$=$

$=$

0

$=$

$\overline{\bar{\sigma}}$

$\overline{\overline{\boldsymbol{\nabla}}}$

5

$\bar{E}$

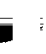




\title{
System Characterization of Positive Real Conditions 1
}

\author{
Q. Wang ${ }^{2}$, J.L. Speyer ${ }^{3}$, and H. Weiss ${ }^{4}$
}

\begin{abstract}
Necessary and sufficient conditions for positive realness in terms of state space matrices are presented under the assumption of complete controllability and complete observability of square systems with independent inpoes. As an altemative to the positive real lemma and to the s-domain inequalities, these conditions provide a recursive algorithm for testing positive realness which result in a set of simple algebraic conditions. By relating the positive real property to the associated variational problem, the paper outlines a unified derivation of aecessary and sufficient conditions for opcimality of boch singular and nonsingular problems.
\end{abstract}

\section{Introduction}

Positive real systems play a major role in control theory. especially in adaptive coatrol, and in stability analysis. The impressive developmeat of adaptive control and self-numing regulation over the last two decades $[1,2]$ is hinged on satisaction of some positive realness conditions. Alternarively, considerable initial knowledge abour the controlled plant must be given. The prior knowledge is used to implement reference models, identifiers, or observer-based controllers of about the same order as the plant. Since the prior assumptions about the controlled plant may never be entirely satisfied, the stability properties of the related adaptive schemes are debatable. Therefore, a direct adaptive control procedure which does not use identifier or observer-based controllers in the feedbact loop is preferred. The implementation of such an algorithm requires positive real controlled plants or altematively, a synthesis of a positive real plant on the basis of the actual plant.

1. This work was parially sponsored by Eglin AFB under Contract F08635-87. K04 17 and by NASA Johrsoo Space Cealer through the RICIS Program of the University of Houscon as Clear Lake.

2. Deparment of Aerospace Engineering and Enginecring Mectarics. University of Texas. Austin. TX 78712

3. Mochanical, Aerospace and Nuclear Engincering Deparment, University of Califomia. Los Anzeles. CA 90024.1597

4. RAFAEL P. O. B. 2250. Huig Isreal
The existing tools for analysis and synchesis of positive real systems are based in the s-domain on complex variable inequalities which are inconvenient or in the state space requiring the positive real lemms equations. These tools are computationally complex and there is a need for an easily used complementary rool. In Secrions 2 and 3, necessary and auficient conditions for positive real systems with independent inputs are developed using optimal control theory for the associated parially singular problem. It is shown that in the totally singular case. these conditions are consistent with the generalized Legendre-Cebsch condition $[3,4]$. The new conditions are associated with the state space matrices of a minimal realization of a square system. The resuling tes for positive realness reduces to recursively lesting cerain square matrices for positive definiteness and the solution to an a gebraic Riccati equation. As an immediate result of the new necessary and sufficient conditions, we also show that the zeros of a positive real system lie in the closed left half complex plane. Some examples are given in Section 4 to illusurate the theory. Concluding marks are given in Section 5.

The derivation of the above results is related to dissipative systems. Basic definitions and physical characteristics are presented below.

\subsection{Dissipative System}

Consider the system input-output description $\mathrm{H}: \mathrm{U} \rightarrow \mathrm{Y}$ where $U=L_{2}^{\prime}\left(R_{+}\right)$and $Y=L_{2}^{\prime}\left(R_{+}\right)$. The notation $L_{2}^{\prime}\left(R_{+}\right)$is used to denote the space of square integrable functions $f: R_{+} \rightarrow R^{\prime}$ where $\mathbf{R}_{+}=[10, \infty)$. The supply rate associated with this system is defined as a function $w: \mathbf{R}^{\prime} \times \mathbf{R}^{m} \rightarrow \mathbf{R}$ where

$$
w(u, y)=y^{\prime} Q y+2 y^{\prime} S u+u^{\prime} R u
$$

and $Q \in \mathbf{R}^{m \boldsymbol{m}} . S \in \mathbf{R}^{\mathrm{md}} . \mathbf{R} \in \mathbf{R}^{\mathbf{L l}}$ are constant matrices, with $Q$ and $R$ symmetric.

Definition 1.1 [5]: A dynamical system $\mathrm{H}$ is dissiparive with respect to the supply rate $w(u, y)$ if and only if

$$
\int_{0}^{l_{1}} w[u(t), y(t)] d t \geq 0
$$

for all $t_{1} \geq w_{0}$ and all $u \in L_{2}^{\prime}$, whenever the initial siste salisfies $x\left(\varphi_{0}\right)=0$. 
Remark 1.1: Eassivity corresponds to dissipativeness where $Q=R=0.1=m . S=\frac{1}{2} I_{m a}$ and $I_{m}$ is mam identity matrix.

Remark 1.2: Rositive realness corresponds to passivity where the dynamical systern is linear and ime invoriant.

Remark 1.3: The concept of a supply rate is relaed in the general case to the "stored energy" for the system As an eximple. suppose that ine system under consideration is an electrical network. whose elements are constants, and $y(t)$ the vector of corresponding port voltage. Then the system is dissipative with respect to the supply rate $w(u, y)=u^{\prime} y$ provided that all the resistances, inductances and capacitance are non-negative.

\subsection{Energy, Power and Information Relationships in}

Dissipative Systems

The class of dissipative systems which has finite dimensional intemal state is completely described in terms of energy storage and power dissipation. Considering this class, the vorious facets of the standard state spuce model can be associated with the concepts of energy, power and information.

Assume that the system under consideration is described by a linear, time-invariant system

$$
\begin{aligned}
& x=A x+B u \\
& y=C x+D u
\end{aligned}
$$

where $x \in R^{n}$, $u \in R^{l}, y \in R^{m}$ and $A, B, C$ and $D$ are constant matrices with appropriate dimension. Then, following [6], the system marices can be regarded as ropresenting:

1. an energy-transformation and dissipation map, ssociated with the mavix $A$.

2. a power injection map, associated with the murices $B$ and $\mathrm{D}$.

3. an information-extraction map, ussociated with the matrix C

Figure 1 describes the-energy-power-infarmation maps associated with the system matrices.

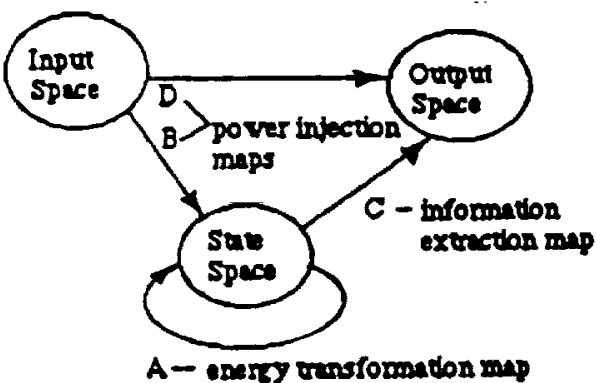

Fig. 1 Energy-Power-Information maps associaled with the System Matrices

The matrix $B$ represents the input coupling between the information represented by the applied input signals and the power available for injection into the system states. The matrix $C$ represents the output coupling between the energy in the system states and the information in the available ourput signals. The macrix $D$ represents the output coupling belween the information represented by the applied input signals and the injected power into the available ourput signals.

\subsection{Review of the Positive Real Property}

The positive real property is related direcily to the iransfer function matrix description of the system. The positive real lemma, presented in Section 2, connects the positive realness to the parameters of a system realization with complete contro!lability and complete observability.

The Positive Real Property [7]: Let $G(s)$ be an $m \times$ in matrix of functions of a complex variable s. then $G(s)$ is termed positive real if the following conditions are satisfied:

(i) All the elements of $\mathrm{G}(\mathrm{s})$ are analytic in $\operatorname{Re}[\mathrm{s}]>0$.

(ii) $G(s)$ is real for real positive $s$.

(iii) $G^{*}(s)+G(s) \geq 0$ for $\operatorname{Re}[s]>0$.

where $(.)^{*}$ denotes complex conjugate transpose.

Remark 1.4: If $G(s)$ is a real rational matrix of functions of s, then necessary and sufficient conditions for the positive real property to hold are given by the following theorem.

Theorem 1.I [7]: Let $G(s)$ be a real rational matrix of functions of \&. Then, $G(s)$ is positive real if and only if:

(i) No element of $G(s)$ has a pole in Re[s] $>0$.

(ii) $G^{*}(j \omega)+G(j \omega) \geq 0$ for all real $\omega$, with $j \omega$ not a pole of any element of $\mathrm{G}(\mathrm{s})$.

(iii) If j $\omega_{0}$ is a pole of any element of $G(s)$, it is at most a simple pole, and the residue matrix.

$$
\begin{aligned}
& k_{0}=\lim _{s \rightarrow j \omega_{0}}\left(s-j \omega_{0}\right) G(s) \quad \text { if } j \omega_{0} \text { is finite, } \\
& k_{-}=\lim _{s \rightarrow j \omega_{0}} G(s) / s \quad \text { if } j \omega_{0} \text { is infinite, }
\end{aligned}
$$

is nonnegative definite Hermitian.

Following Definition 1.1, if the system is positive real, the angle between the output vector $y(t)$ and the input vector $u(t)$ is bounded below by -90 deg. and above by +90 deg.

\section{Relations Between Optimal Control and Positive Realness}

\subsection{The Related Variational Problem \\ Consider the coss functional}

$$
V\left[x_{0}, t a, u(\cdot)\right]=\int_{t_{0}}^{t_{1}} w(u(t), y(t)] d t
$$

where the supply rate

$$
w(u, y)=y^{\prime} u=u^{\prime} D^{\prime} u+x^{\prime} C u
$$

is associated with system (1.3) and (1.4), where the dimensions of $u$ and $y$ are $m$. The problem is to find necessary and sufficient conditions for optimality of $u^{*}(\cdot) \in U$ to minimize $V\left(x_{0}, t_{0}, u(\cdot)\right)$. 
denoted $V *\left[x_{0}, t_{0}\right]$, subject to the dynamic equation of (1.3) where $x\left(L_{0}\right)=x_{0}$ is prescribed.

Remark 2.1: Since only the symmetric part of D contributes $10 w(u, y)$, then

$$
w(u, y)=\frac{1}{2}\left(u^{\prime} R u+2 x^{\prime} C u\right)
$$

where

$$
R=D+D^{\prime}
$$

Remark 2.2: If $R \geq 0$, and $\operatorname{rank}(R)=r<m$, there exists an orthogonal transformation $\Gamma=\left[\Gamma_{1}, \Gamma_{2}\right]$ such that

$$
\left[\begin{array}{l}
\Gamma_{1} \\
\Gamma_{1}
\end{array}\right] R\left[\Gamma_{1}, \Gamma_{2}\right]=\left[\begin{array}{cc}
R_{1} & 0 \\
0 & 0
\end{array}\right]
$$

where $R_{p}$ is positive. For instance, $\Gamma_{1}$ and $\Gamma_{2}$ may consist of nomalized eigenvectors of $\mathbf{R}$ associated with nonzero and zero eigenvalues, respectively [8]. There is a narural partitioning of the control vector associated with this transformation, a r-dimensional nonsingular control and an (m-r)-dirbensional singular control

\subsection{Positive Real Lemma Equations}

Necessary and sufficient condition for $V^{*}\left[x_{0}, t_{0}\right]$ to be bounded below over a finite time interval $\left[t_{0}, b_{1}\right]$ are presented in Theorem II 3.3 of [9]. The required positive real conditions are obtained via the extension of the optimality condition to the timeinvariant, infinite-time case [10].

Under the complete controllability and complete observability assumption of system (1.3), necessary and sufficient conditions for the nonnegativity of $V[0,6, u(\cdot)]$ are that there exist $\pi<0, L$, and W such that

$$
\left[\begin{array}{cc}
\pi A+A^{\prime} \pi & \pi B^{\prime}+C^{\prime} \\
B^{\prime} \pi+C & R
\end{array}\right]=\left[\begin{array}{l}
L^{\prime} \\
W^{\prime}
\end{array}\right][L, W] \geq 0 .
$$

where $W$ and $L$ are matrices with proper dimension.

By identifying $P=-\pi$, the positive real Lemma is stated.

The Positive Real Lemma [7]: Let $G(s)$ be an $m \times m$ marrix of real rational functions of a complex variable $s$, with $G(\infty)<\infty$. Let $(A, B, C, D\}$ be a minimal realization of $G(s)$. Then, $G(s)$ is positive real if and only if there exist real maurices $P$, $L$, and $W$ with $P$ positive definite and symmetric, such that:

$$
\begin{aligned}
& P A+A^{\prime} P=-L Z \\
& B P=C-W L \\
& W W=D+D^{\prime}
\end{aligned}
$$

Remark 2.2: The generalized Legendre-Clebsch condition, which is a necessary condition for $V *\left[x_{0}, 10\right]>-\infty$ in the totally singular case, given in [3] for a linear time-invariant system can be written as

$$
\begin{aligned}
& \frac{\partial}{\partial u}\left(\dot{H}_{u}\right)=C B-(C B)^{\prime}=0 \\
& \frac{\partial}{\partial u}\left(\dot{H}_{u}\right)=C A B+(C A B)^{\prime} \leq 0
\end{aligned}
$$

where $H$ is the variational Hamiltonian and $\lambda \in \mathbf{R}^{n}$ is the associared Lagrange multiplier

$$
H=u^{\prime} C x+\lambda^{\prime}(A x+B u), \quad \dot{\lambda}=-H_{x} .
$$

By lerting $R=0$, the necessary conditions (2.10) and (2.11) are also obrained from the positive real kemma.

\section{Positive Real Conditions in Terms of State-Space Matrices}

Necessary and sufficient conditions for the nonnegativity of $V[0,6, u(\cdot)]$ are given by the existence of $\pi<0, L$, and $W$ which satisfy (2.6). Let $G(s)$ be an $m \times m$ matrix of degree $n$. Consider a minimal realization (A, B, C, D) representing the finitediniensional linear time-invariant dynamic equations given by (1.3) and (1.4). In terms of state space matrices A, B, C, and D, (2.6) gives necessary and sufficient conditions for positive real system In this section. new necessary and sufficient conditions are developed.

\subsection{Standard Formulation of the Partially Singular Problem}

Assume that $G(s)$ is a square matrix of proper rational function with independent columns. For any realization, the matrices $C$ and $B$ are full rank. Without loss of generality, we consider a minimal realization $(A, B, C, D$ ) of the form that $A$ is an nxn matrix which is partitioned as

$$
A=\left[\begin{array}{ll}
A_{11} & A_{12} \\
A_{21} & A_{22}
\end{array}\right] \text {. }
$$

where $A_{11}$ is a bxk matrix, and $A_{22}$ is an $(n-k) \times(n-k)$ matrix, where $k$ is the dimension of the singular control.

$$
B=\left[\begin{array}{ll}
B_{r}, & B_{3}
\end{array}\right], \quad C=\left[\begin{array}{l}
C_{1} \\
C_{2}
\end{array}\right] \text {. }
$$

and

$$
D+D^{\prime}=\left[\begin{array}{cc}
R, & 0 \\
0 & 0
\end{array}\right]
$$

where $R_{t}$ is a $r x r$ nonsingular matrix corresponding to the nonsingular control, $B_{r}$ is an axr maurix, $B_{z}$ is an $n \times k$ matrix related to the singular control, $C_{l}$ is a ren matrix, and $C_{3}$ is a $k \times n$ matrix, where $r=m-k$ is the dimension of the nonsingular control. If $n>k$, then $C_{3}$ has the following form

$$
C_{1}=\left[C_{11}, 0\right] \text {. }
$$

where $C_{1}$ is a nonsingular matrix. Correspondingly. $B_{1}$ is written as $\left[\begin{array}{l}B_{31} \\ B_{32}\end{array}\right]$. We define this as a standard realization.

Notice that the realization can be obtained by choosing suitable bases for the staic space and the inpuloutput space. For example, suppose $\{\bar{A}, \bar{B}, \bar{C}, G(\infty)\}$ is a minimal realization of $G(s)$. Let the column vectors of $\Gamma$, where $\Gamma$ is described in Remark 2.2, be a basis of the inpuVoutput space, then the following cansformation $y=\Gamma \eta, u=\Gamma v$ is defined. Furthermore, let $q_{1}, q_{2}, \ldots, q_{n}, t, q_{n-k+1}, \ldots, q_{n}$ be a basis of the state space, where $q_{n-k+1}, \ldots, q_{n}$ span the null space of $r_{2} \bar{C}$, and $q_{1}, q_{2}, \ldots$, $q_{n \cdot k}$ are arbitrary vectors such that $Q=\left[q_{1}, q_{2}, \ldots, q_{n \cdot k}, q_{n \cdot k+1}\right.$, $\left.\ldots, q_{n}\right]$ is nonsingular. This defines a transformation $x=Q_{3}$. The resulting dynamic equacion can be writen as

$$
\begin{aligned}
& \dot{\xi}=A \xi+B v \\
& \eta=C \xi+D v,
\end{aligned}
$$

where $A=Q^{\cdot} \cdot \bar{A} Q, B=Q \cdot 1 \bar{B} \Gamma, C=\Gamma \cdot \bar{C} Q$, and $D=\Gamma G(\infty) \Gamma$. The transfer function marix of this system is $\Gamma G(s) \Gamma$. the positive 
realness of $\mathrm{G}(\mathrm{s})$ is equivalent to the positive realness of $\Gamma \mathrm{G}(\mathrm{s}) \Gamma$. The application of (2.6) and development of the new necessary and sufficient conditions for the partially singular problem will be discussed under assumption of a standard realization as discussed.

\subsection{Derivation of New Necessary and Sufficient Conditions}

Necessary and sufficient condition for nonnegative of $V[0,10 . u(\cdot)]$ as given by condition (2.6) can be restated in the following equivalent forms: There exist a $\pi<0$ and a matrix $V$ such that

$$
\left[\begin{array}{cc}
\pi A+A^{\prime} \pi & \pi B+C^{\prime} \\
B^{\prime} \pi+C & R
\end{array}\right]=V^{\prime} V
$$

Furthermore, $R$ being positive semi-definite is a necessary condition for satisfying (3.3). If $R>0$, then (3.3) can be reduced to a condition based upon a Recati equation. That is, there exists a negative definite solution $\pi$ to the algebraic Ricati equation

$\pi\left(A-B^{-1} C\right)+\left(A-B R^{-1} C\right)^{\prime} \pi-\pi B^{-1} B^{\prime} \pi \cdot C^{-1} C=0$.

If $R$ is singular, (3.3) can be written as

$$
\left[\begin{array}{ccc}
\pi A+A^{\prime} \pi & \pi B_{r}+C_{r}^{\prime} & \pi B_{s}+C_{s} \\
B_{r}^{\prime} \pi+C_{r} & R_{r} & 0 \\
B_{r}^{\prime} \pi+C_{s} & 0 & 0
\end{array}\right]=V V
$$

or, equivalently, there exist a $\pi<0$ and a matrix $V_{r}$ auch that

$$
\pi B_{3}+C_{i}^{\prime}=0
$$

and

$$
\left[\begin{array}{cc}
\pi A+A^{\prime} \pi & \pi B_{r}+C_{t}^{\prime} \\
B_{r}^{\prime} \pi+C_{r} & R_{r}
\end{array}\right]=V_{r} V_{r} .
$$

If the dimension of the state is less than or equal to the dimension of the singular control, $L_{2} n \leq k, \pi$ can be determined from equation (3.5). If and only if $2 x<0$ is solvable from (3.5) and the same $\pi$ satisfies (3.6), the system is positive real. If $n>$ $k$. the fact $\pi<0$ and equation (3.5) iruply that

$$
C_{3} B_{3}=\left(C_{2} B_{3}\right)^{\prime}=-B_{3}{ }^{\prime} \times B_{3}>0 \text {. }
$$

Since $C_{8}=\left[C_{11}, 0\right]$, and $C_{71}$ is monsingular. Equation (3.7) also implies that $B_{11}$ is nonsingular. Furthermore (3.5) provides a linear constraint on $x$ which is discussed in Lemm 3.1 below.

Lemma 3.1: $\pi<0, \pi B_{8}+C_{3}^{\prime}=0$ if and only if $C_{3} B_{3}$ $>0$ and

$$
\pi=\left[\begin{array}{cc}
-\left(B_{11}\right)^{-1} C_{21}+\left(B_{11}\right)^{-1} B_{12} \pi_{1} B_{22}\left(B_{31}\right)^{-1} & -\left(B_{31}\right)^{-1} B_{32} \pi_{1} \\
-\pi_{1} B_{12}\left(B_{31}\right)^{-1} & \pi_{1}
\end{array}\right]
$$

for some $\pi_{1}<0$.

$$
\text { Proof: Denore } \pi \text { as } \pi=\left[\begin{array}{ll}
\pi_{11} & \pi_{12} \\
\pi_{12} & \pi_{1}
\end{array}\right] \text {. To prove }
$$

sufficiency, we assume that $\pi_{1}<0, C_{3} B_{3}>0$, and

$$
\begin{aligned}
& \pi_{11}=-\left(B_{31}\right)^{\prime} C_{31}+\left(B_{11}\right)^{-1} B_{32} \pi_{1} B_{32}\left(B_{31}\right)^{-1} \\
& \pi_{12}=-\left(B_{31}\right)^{-1} B_{32} \pi_{1} .
\end{aligned}
$$

Define $F=\left[\begin{array}{cc}1 & -\pi_{12}\left(\pi_{1}\right)^{-1} \\ 0 & 1\end{array}\right]$, then $F$ is nonsingular and

$$
\begin{aligned}
F \pi F & =\left[\begin{array}{cc}
I & -\pi_{12}\left(\pi_{1}\right)^{-1} \\
0 & I
\end{array}\right]\left[\begin{array}{cc}
\pi_{11} & \pi_{12} \\
\pi_{12} & \pi_{1}
\end{array}\right]\left[\begin{array}{cc}
1 & 0 \\
-\left(\pi_{1}\right)^{-1} \pi_{12} & 1
\end{array}\right] \\
& =\left[\begin{array}{cc}
\pi_{1}-\pi_{12}\left(\pi_{1}\right)^{-1} \pi_{12} & 0 \\
0 & \pi_{1}
\end{array}\right]=\left[\begin{array}{cc}
-\left(B_{11}\right)^{-1} C_{31} & 0 \\
0 & \pi_{1}
\end{array}\right] .
\end{aligned}
$$

Since $C_{8} B_{2}=C_{11} B_{11}>0$,

$$
-\left(B_{31}\right)^{-1} C_{81}=-\left(B_{3 i}\right)^{-1} C_{81} B_{3}\left(B_{31}\right)^{-1}<0 \text {. }
$$

Therefore $F \pi F<0$, and it also implies that $\pi<0$. Furthermore. by using $\pi_{11}$ and $\pi_{12}$ defined in (3.9) and (3.10), we get

$$
\pi B_{3}+C_{6}^{\prime}=\left[\begin{array}{l}
\pi_{11} B_{11}+\pi_{12} B_{32} \\
\pi_{12} B_{31}+\pi_{1} B_{s 2}
\end{array}\right]+\left[\begin{array}{c}
C_{11} \\
0
\end{array}\right]=\left[\begin{array}{l}
0 \\
0
\end{array}\right] \text {. }
$$

Next, we prove the necessity. If $\pi<0$, then $\pi_{1}<0$. From $\pi B_{s}+$ $C_{s}^{\prime}=0$ we get

$$
\begin{aligned}
& \pi_{11} B_{s 1}+\pi_{12} B_{s 2}+C_{s 1}{ }^{\prime}=0 \\
& \pi_{12} B_{s 1}+\pi_{1} B_{s 2}=0
\end{aligned}
$$

By solving (3.12) and (3.13), the expressions of $\pi_{11}$ and $\pi_{12}$ are obtained which are the same as shown in equations (3.9) and (3.10).

Q.E.D.

Let the matrix shown in (3.6) be denoted as $M\left(\pi, R_{r}\right)$

$$
M\left(\pi, R_{r}\right)=\left[\begin{array}{cc}
\pi A+A^{\prime} \pi & \pi B_{r}+C_{r}^{\prime} \\
B_{r}^{\prime} \pi+C_{r} & R_{r}
\end{array}\right] .
$$

For any nonsingular matrix $T,(3.6)$ is equivalent to $T M\left(\pi, R_{r}\right) T$ $=V_{T} V_{T}$, where $V_{T}$ is a murix with proper dimension. By defining

$$
T_{r}=\left[\begin{array}{ccc}
0 & B_{11} & 0 \\
1 & B_{s 2} & 0 \\
0 & 0 & I
\end{array}\right]
$$

and using $\pi$ defined in (3.8) as a function of $\pi_{1}$, then $T_{1}$ is nonsingulur, and

$$
T_{r} M\left(\pi, R_{1}\right) T_{2}=\left[\begin{array}{lll}
M_{11} & M_{12} & M_{12} \\
M_{12} & M_{22} & M_{23} \\
M_{13} & M_{23} & M_{33}
\end{array}\right]
$$

where

$$
\begin{aligned}
& M_{11}=\left[\begin{array}{lll}
0 & {[} & 0
\end{array}\right] M(\pi, R)\left[\begin{array}{l}
0 \\
1 \\
0
\end{array}\right] \\
& =\pi_{1}\left(A_{22}-B_{32}\left(B_{31}\right)^{-1} A_{12}\right)+\left(A_{22}-B_{32}\left(B_{31}\right)^{-1} A_{12}\right)^{\prime} \pi_{1} \\
& M_{12}=\left[\begin{array}{lll}
0 & 1 & 0
\end{array}\right] M(\pi, R)\left[\begin{array}{c}
B_{11} \\
B_{22} \\
0
\end{array}\right]=\pi_{1}\left(A_{21} B_{31}+A_{22} B_{32}\right. \\
& \left.B_{32}\left(B_{31}\right)^{-1} A_{11} B_{31}-B_{22}\left(B_{31}\right)^{-1} A_{12} B_{32}\right) \cdot C_{11} A_{12} \\
& M_{22}=\left[B_{31}, B_{32}, 0\right] M(\pi, R)\left[\begin{array}{c}
B_{31} \\
B_{12} \\
0
\end{array}\right] \\
& =-\left(C_{3} A B_{3}+B_{3} A^{\prime} C_{3}\right)
\end{aligned}
$$

$$
\begin{aligned}
M_{23} & =\left[B_{1} 1^{\prime}, B_{32} ; 0\right] M(\pi, R)\left[\begin{array}{l}
0 \\
0 \\
1
\end{array}\right] \\
& =-C_{3} B_{r}+B_{2} C_{t} \\
M_{33} & =[0.0 .1] M(\pi, R)\left[\begin{array}{l}
0 \\
0 \\
1
\end{array}\right]=R_{f} .
\end{aligned}
$$


By defining

$$
\begin{aligned}
& A_{1}=A_{22} \cdot B_{52}\left(B_{11}\right)^{-1} A_{12} \\
& B_{1}=\left[A_{21} B_{31}+A_{22} B_{32}-B_{32}\left(B_{31}\right)^{-1} A_{11} B_{31}\right. \text { - } \\
& \left.B_{12}\left(B_{11}\right)^{-1} A_{12} B_{22}, B_{12}\right] \\
& C_{1}=\left[\cdot C_{11} A_{12}, 0\right]
\end{aligned}
$$

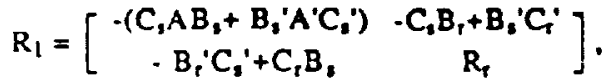

a condition which is equivalent to (3.6) can be stated as the follows: There exist a $\pi_{1}<0$ and a marrix $V_{1}$ such that

$$
\left[\begin{array}{cc}
\pi_{1} A_{1}+A_{1}^{\prime} \pi_{1} & \pi_{1} B_{1}+C_{1}^{\prime} \\
B_{1}^{\prime} \pi_{1}+C_{1} & R_{1}
\end{array}\right]=V_{1} V_{1}
$$

According to the positive real lemma, Equation (3.18) implies that $\left\{A_{1}, B_{1}, C_{1}, \frac{R_{1}}{2}\right\}$ is positive real.

\subsection{Necessary and Sufficient Conditions for Positive Realness}

The results in Section 3.2 are summarized in the next theorem as an altemarive necessary and sufficient condition for testing positive realness of a square system.

Theorem 3.1: The necessary and sufficient condition for (A, B, C, D) to be positive real is that

(i) $\mathrm{R} \geq 0$;

(ii) If $R>0$, there exists a positive definite solution $P$ to the following algebraic Riccati equation $\mathbf{P}\left(\mathrm{A}-\mathrm{BR}^{-1} \mathrm{C}\right)+\left(\mathrm{A}-\mathrm{BR}^{-1} \mathrm{C}\right) \mathbf{P}+\mathbf{P} \mathbf{B R}^{-1} \mathbf{B} \mathbf{P}+\mathrm{CR}^{-1} \mathbf{C}=0$;

(iii) If rank $R=r<m$, and $n \leq m-r$, there exists $P=C_{3} B_{3}^{\prime}\left(B_{3} B_{3}^{\prime}\right)^{-1}>0$ satisfying $P B_{3}=C_{3}$ and

$$
\left[\begin{array}{cc}
-P A-A^{\prime} P-P B_{r}+C_{t}^{\prime} \\
-B_{t}^{\prime} P+C_{t} & R_{t}
\end{array}\right] \geq 0
$$

(iv) If rank $R=r<m$, and $n>m-r$, then $\left.C_{1} B_{1}=\left(C_{2} B_{2}\right)^{\circ}\right\rangle$ 0 and $\left\{A_{1}, B_{1}, C_{1}, \frac{R_{1}}{2}\right\}$ is positive real, where $A_{1}, B_{1}, C_{1}$, and $R_{1}$ are defined in equations (3.14) to (3.17).

Condition (ii) is obtained by identifying $P$ with $-\pi$ in equation (3.4). Condition (iii) is the interpretacion of (3.5) and (3.6) for the case $n \leq(m-r)$. If $P=\pi>0$ exists, then $P B_{3}=C_{i}$ : $\mathrm{PB}_{\mathrm{s}} \mathrm{B}_{\mathrm{s}}{ }^{\prime}=\mathrm{C}_{\mathrm{s}} \mathrm{B}_{\mathrm{s}}$, and $\mathrm{P}=\mathrm{C}_{1} \mathrm{~B}_{\mathrm{s}}{ }^{\prime}\left(\mathrm{B}_{\mathrm{s}} \mathrm{B}_{\mathrm{s}}\right)^{-1}>0$. Condition (iv) corresponds to the sinuation we discussed through (3.7) to (3.13)

Remark 3.1: Alternative transformution approaches to the singular problem using the Kelley transformation for the linear quadratic problem are given in [9] for the matrix case. The approach here is different via the suncture of $x$ given by Lemma 3.1.

Remark 3.2: If (A, B, C, D) is a minimal realization. then it is required for a positive real system that there exists a positive definite mavix $P$ such that

$$
P A+A P \leq 0
$$

Therefore. it is required that $\operatorname{Re} \lambda_{i}[A] \leq 0$ and the Jordan form of $A$ has no blocks of size greater than $|x|$ with pure imaginary diagonal elements.

Remark 3.3: If $G(s)$ is sirictly proper, the minimal realization is totally singular, then the characteristic polynomial of
$A_{1}=A_{22} \cdot B_{32}\left(B_{11}\right)^{1} A_{12}$ is equal to the zero polynomial of the system up to a nonzero scalar factor.

Proof: Let $\operatorname{det} G(s)=\operatorname{det}\left(C(I s-A)^{\cdot} B\right)=\frac{\psi(s)}{S(s)}$, where

$$
\Delta(\mathrm{s})=\operatorname{det}(\text { Is - A ) }
$$

and $\Psi(s)$ is the zero polynomial of the system. Since state feedbaks do not change the numerator of the transfer function matrix, for any matrix $\mathrm{K}$.

$$
\operatorname{det}\left(G_{k}(s)\right)=\operatorname{det}\left(C\left(I_{5}-A-B K\right)^{-1} B\right)=\frac{\psi(s)}{\Delta_{k}(s)}
$$

where $\Delta_{k}(s)=\operatorname{det}(I s-A \cdot B K)$

Let $K=\left[0,\left(B_{11}\right)^{-1} A_{12}\right]$, then

$A+B K=\left[\begin{array}{cc}A_{11} & 0 \\ A_{21} & A_{22}-B_{32}\left(B_{31}\right)^{-1} A_{12}\end{array}\right]=\left[\begin{array}{cc}A_{11} & 0 \\ A_{21} & A_{1}\end{array}\right]$

$$
\begin{aligned}
\Delta_{k}(s) & =\operatorname{det}(I s-A-B K)=\operatorname{det}\left[\begin{array}{cc}
I s-A_{11} & 0 \\
-A_{21} & I s-A_{1}
\end{array}\right] \\
& =\operatorname{det}\left(I s-A_{11}\right) \operatorname{det}\left(I s-A_{1}\right)
\end{aligned}
$$

$$
\begin{aligned}
\operatorname{det}\left(G_{k}(s)\right) & =\operatorname{det}\left(C\left(I_{s}-A-B K\right)^{-1} B\right)=\operatorname{det}\left(C_{31}\left(I s-A_{11}\right)^{-1} B_{s 2}\right) \\
& =\frac{\operatorname{det}\left(C_{s 1}\right) \operatorname{det}\left(B_{31}\right)}{\operatorname{det}\left(I s-A_{11}\right)}
\end{aligned}
$$

Therefore,

$$
\begin{array}{r}
\Psi(s)=\Delta_{k}(s) \operatorname{det}\left(G_{k}(s)\right)=\operatorname{det}\left(C_{31}\right) \operatorname{det}\left(B_{s 1}\right) \operatorname{det}\left(I s-A_{1}\right) \\
\text { Q.E.D. }
\end{array}
$$

Remark 3.4: From (3.18) and Remark 3.3, we conclude that there are $\mathrm{n}$ - $\mathrm{m}$ finite zeros for a positive real system and all the zeros lie in the closed left half complex plane. In other words, the system is minimum phase.

\section{Examples}

Theorem 3.1 introduces a recursive procedure for testing positive real systems, requests only for testing a series of matrices $C_{i s} B_{i s}>0$, for $i=0,1,2, \ldots, l$, and the solution to a algebraic Riccati equation $P_{1}>0$, where $i$ is the index associated with the new system obtained from the $i$-th iteration, and $i=0$ corresponds to $B_{3}, C_{1}$, and $P$. The testing stops when $R_{1}$ becomes nonsingular, or the dimension of the state is less or equal to the dimension of the singular control.

The following examples illustrate the application of Theorem 3.1 .

Example 4.1: Given $G(s)=\frac{(s+2)^{2}}{s(s+1)(s+3)}$, an observable realization of $\mathbf{G}(\mathrm{s})$ is

$$
A=\left[\begin{array}{ccc}
-4 & 1 & 0 \\
-3 & 0 & 1 \\
0 & 0 & 0
\end{array}\right], \quad B=\left[\begin{array}{l}
1 \\
4 \\
4
\end{array}\right], \quad C=[1,0,0], \quad D=0
$$

Furst iteration:

$\mathbf{R}=\mathbf{0}$

$\mathrm{CB}=(\mathrm{CB})^{r}=1>0$ 
$A_{1}=\left[\begin{array}{ll}-4 & 1 \\ -4 & 0\end{array}\right], \quad B_{1}=\left[\begin{array}{l}1 \\ 0\end{array}\right], \quad C_{1}=[-1,0] . \quad R_{1}=0$

Second ileration:

$R_{1}=0$

$C_{1} B_{1}=\left(C_{1} B_{1}\right)^{\prime}=-1<0$

Therefore, the system is not posioive real.

Example 4.2: Given $G(s)=\frac{(s+1)^{2}}{s(s+2)(s+4)}$, an observable realization of $\mathrm{G}(\mathrm{s})$ is

$$
\begin{array}{rl}
A= & {\left[\begin{array}{ccc}
-6 & 1 & 0 \\
-8 & 0 & 1 \\
0 & 0 & 0
\end{array}\right], \quad B=\left[\begin{array}{l}
1 \\
2 \\
1
\end{array}\right], \quad C=[1,0,0], \quad D=0 .} \\
& \text { First itcaion: } \\
R=0 & C B=(C B)^{\prime}=1>0 \\
& A_{1}=\left[\begin{array}{cc}
-2 & 1 \\
-1 & 0
\end{array}\right], \quad B_{1}=\left[\begin{array}{l}
1 \\
4
\end{array}\right], \quad C_{1}=[-1,0], \quad R_{1}=8 .
\end{array}
$$

Second iteration:

$R_{1}=8>0$, the algebraic Riccati equation is

$$
\begin{aligned}
P_{1}\left[\begin{array}{cc}
-15 & 8 \\
-4 & 0
\end{array}\right] & +\left[\begin{array}{cc}
-15 & -4 \\
8 & 0
\end{array}\right] P_{1}+ \\
& +P_{1}\left[\begin{array}{cc}
1 & 4 \\
4 & 16
\end{array}\right] P_{1}+\left[\begin{array}{ll}
1 & 0 \\
0 & 0
\end{array}\right]=0
\end{aligned}
$$

which has a positive definite solution

$$
P_{1}=\left[\begin{array}{cc}
0.0394 & -0.0225 \\
-0.225 & 0.1557
\end{array}\right]>0
$$

Therefore, the system is positive real.

Example 4.3: $\quad G(s)=\frac{s^{2}+z^{2}}{s\left(s^{2}+p^{2}\right)} \cdot \quad$ A minimal ratization of $G(s)$ is

$$
A=\left[\begin{array}{ccc}
0 & 1 & 0 \\
-p^{2} & 0 & 1 \\
0 & 0 & 0
\end{array}\right], \quad B=\left[\begin{array}{l}
1 \\
0 \\
z^{2}
\end{array}\right] . \quad C=[1,0,0], \quad D=0 .
$$

First itration:

$R=0$

$\mathrm{CB}=(\mathrm{CB})^{\prime}=1>0$

$A_{1}=\left[\begin{array}{cc}0 & 1 \\ -z^{2} & 0\end{array}\right], \quad B_{1}=\left[\begin{array}{c}z^{2} \cdot p^{2} \\ 0\end{array}\right], \quad C_{1}=\{-1,0\}$.

$\mathbf{R}_{1}=0$.

Second iteration:

$R_{1}=0$.

$C_{1} B_{1}=\left(C_{1} B_{1}\right)^{\prime}=p^{2} \cdot z^{2}>0$ if and only if $p^{2}>z^{2}$

$A_{2}=0, \quad B_{2}=-2^{2}\left(z^{2} \cdot p^{2}\right), \quad C_{2}=1, \quad R_{2}=0$.

Third iteration:

$\mathrm{R}_{2}=0$

$P_{2}=C_{2} B_{2}\left(B_{2} B_{2}\right)^{-1}=\frac{1}{z^{2}\left(p^{2} \cdot z^{2}\right)}>0$ if $p^{2}>z^{2}$

$-P_{2} A_{2}-A_{2} \cdot P_{2}=0$.

Therefore, the system is positive real if and only if $p^{2}>z^{2}$
5. Summary and Conclusions

This paper reviews positive real system as a subclass of dissipative systems and states the positive real lemma equations. By using the variational problem associated with the parially singular problem, necessary and sufficient conditions for a system to be positive real are derived. These conditions are particularly transparent by using Lemma 3.1 which provides a uniquely structure for the matrix $\pi$. These positive realness conditions are expressed in terms of the state space matrix inequalities and algebraic Riccati equations and do nor deal with inequalities in the $s$ domain or with solutions of the positive real lemma equations. These tests are direct, and a system either satisfies these conditions or not. There is no requirement to search over all matrices to determine if a condition can be satisfied as in the positive real lerama. Examples are given which demonstrate the power of this approach.

\section{References}

[1] L. D. Landau, Adoprive Control . The Model Reference Approach, Marcel Dekker, New York, 1979.

[2] K J. Astrom, Theory and Applications of Adaptive Control - A Survey," Automatica, Vol. 19. No. S, pp. 471-481, 1983.

[3] D. H. Jacobson and J. L. Speyer, Necessary and Sufficient Conditions for Optimality for Singular Control Problems: A Limit Approach," J. Marh. Anal. Appl., Vol. 34, No. a May 1971, pp. 139-266.

[4] D. J. Bell and D. H. Jacobson, Singular Optimal Control Problems, Academic Press, New York, 1975.

[5] D. J. Hill and P. J. Moylan, Dissipative Dynamical Systems: Basic Input-Output and State Properties." Jowrnal of the Franklin Institure, Vol. 309. No. 5, May 1980, pp. 327-357

[6] A. G. J. MacFarlane and Karcanias, "Poles and Zeros of Linear Multivariable Systems: A Survey of the Algebraic, Geometric and Complex-Variable Theory," Int. J. Control, Vol. 24, No. 1, 1976, pp. 33-74.

[7] B. D. O. Anderson and Vongpanitlend, Nemork Analysis and Synsheris - A Modern System Theory Approach. Prentice-Hall, Englewood Clifts, New Jersey, 1973.

[8] C. T. Chen, Linear System Theory and Design. Holt, Rinehat and Winston, New York, 1984.

[9] D. J. Clements and B. D. OD. Anderson, Singular Oprimal Contral: The Linear Quadratic Problem. Lecture Nores in Control and Information Sciences edited by A. V. Balakrishnan and $M$. Thoma, Spriner-Verlag, Berlin Heidelberg, New York. 1973.

[10] J. C. Willems, "Least Squares Stationary Optimal Control and the Algebraic Riccati Equation." IEEE Trans. Ausomat. Consr. Vol. AC-16, pp. 621-634, Dec. 1971. 